hep-th/0410093

October 2004

\title{
Ricci flows and their integrability in two dimensions*
}

\author{
Ioannis Bakas ${ }^{\dagger}$ \\ Department of Physics, University of Patras \\ GR-26500 Patras, Greece \\ bakas@ajax.physics . upatras.gr
}

\begin{abstract}
We review the main aspects of Ricci flows as they arise in physics and mathematics. In field theory they describe the renormalization group equations of the target space metric of two dimensional sigma models to lowest order in the perturbative expansion. As such they provide an off-shell approach to the problem of tachyon condensation and vacuum selection in closed string theory in the weak gravitational regime. In differential geometry they introduce a systematic framework to find canonical metrics on Riemannian manifolds and make advances towards their classification by proving the geometrization conjecture. We focus attention to geometric deformations in low dimensions and find that they also exhibit a rich algebraic structure. The Ricci flow in two dimensions is shown to be integrable using an infinite dimensional algebra with antisymmetric Cartan kernel that incorporates the deformation variable into its root system. The deformations of two dimensional surfaces also control the Ricci flow on 3-manifolds and their decomposition into prime factors by applying surgery prior to the formation of singularities along shrinking cycles. A few simple examples are briefly discussed including the notion of Ricci solitons. Other applications to physical systems are also listed at the end.
\end{abstract}

${ }^{*}$ Contribution to the proceedings of the conference Strings '04 held in Paris, 28 June - 2 July 2004; to be published in a special volume of Comptes Rendus de l' Académie des Sciences Paris.

${ }^{\dagger}$ Address from 1 October 2004 to 30 September 2005: Theory Group, Physics Department, CERN, CH-1211 Geneva 23, Switzerland; e-mail: ioannis.bakas@cern.ch. 
The Ricci flow equations arose independently in physics and mathematics in the early '80s. Since then they have become a major tool for addressing a variety of problems in the quantum theory of fields and strings as well as in geometry where some ground breaking results have been obtained in recent years (see, for instance, [1] for a recent collection of important mathematical papers on the subject). The Ricci flows are second order non-linear parabolic differential equations for the components of the metric of an $n$-dimensional Riemannian manifold which are driven by the Ricci curvature tensor,

$$
\frac{\partial}{\partial t} G_{\mu \nu}=-R_{\mu \nu} .
$$

As such they describe geometric deformations of the Riemannian metric with parameter $t$ (called time) that generalize the heat flow equation beyond the weak field approximation $G_{\mu \nu} \simeq \delta_{\mu \nu}+h_{\mu \nu}$, which is only valid for small and slowly varying perturbations of the metric $h_{\mu \nu}$. From the analysis of such partial differential equations one obtains existence and uniqueness theorems of solutions on some time interval starting from any smooth initial metric; in some cases the solutions exist after infinitely long time in the sense that the metric does not become singular anywhere. Our purpose is to review the main properties of these equations and consider some of their current applications in physics and mathematics. It is a hopeless task to find their general solution in arbitrary number of dimensions $n$, since the fixed point configurations $R_{\mu \nu}=0$ are already quite difficult to determine exactly. Fortunately, several important results depend only on the qualitative properties of the flows and not on exact solutions, but many times it is also useful to have explicit expressions, as in the theory of gravitation.

We will first present the general aspects of Ricci flows in arbitrary number of dimensions and then specialize the discussion to low dimensions. It will be shown that the Ricci flow defines an integrable system in two dimensions using a local system of conformally flat coordinates. In this case, the general solution can be obtained by Bäcklund transformations as in Toda field equations associated to simple Lie algebras. A few special solutions will also be described together with their physical interpretation. An important feature of our method is the use of infinite dimensional algebras that incorporate the deformation variable $t$ into their root system. Thus, apart from the ability to linearize the Ricci flow and parametrize all geometric deformations in two dimensions using free fields, we uncover a novel algebraic structure which might be valuable for the reformulation of more general dynamical problems in gravitational theories. Our approach also brings to light the class of Lie algebras with non-symmetrizable Cartan matrices which have been very poorly studied in mathematics and have never been used in physics so far. Finally, we will discuss the relevance of two dimensional flows to the topology of 3-manifolds and summarize some of the recent developments in the geometrization of 3-manifolds via the Ricci flow.

A simple solution that illustrates the main qualitative features of the Ricci flow in all dimensions is derived by considering an initial metric $G_{\mu \nu}(0)$ of constant Ricci curvature $R_{\mu \nu}=a G_{\mu \nu}$. Then, the evolution proceeds by rescaling the metric uniformly in all 
directions, as

$$
G_{\mu \nu}(t)=(1-a t) G_{\mu \nu}(0)
$$

Clearly, the metric has constant Ricci curvature at all later times but its size depends on the sign of $a$; if $a>0$ the metric will contract uniformly and the formation of singularity becomes inevitable as in a big crunch, whereas if $a<0$ the metric will expand smoothly for ever. This simple solution admits a physical interpretation in the renormalization theory of two dimensional sigma models. Consider the $O(N)$ sigma model which is defined by the two dimensional action

$$
S=\frac{1}{g^{2}} \int d^{2} w(\partial \vec{n})^{2}
$$

using an $N$-dimensional unit vector field $\vec{n}$. Its target space is $S^{N-1}=S O(N) / S O(N-1)$ and has positive constant Ricci curvature. The quantum theory is not conformal but it is perturbatively renormalizable with coupling constant $g^{2}$ changing (to lowest order) as follows,

$$
\frac{1}{\tilde{g}^{2}}=\frac{1}{g^{2}}+\frac{N-2}{4 \pi} \log \frac{\tilde{\Lambda}}{\Lambda}
$$

under changes of the world-sheet length scale $\Lambda^{-1}$ (see, for instance, [2]). Setting

$$
t=\log \Lambda^{-1}
$$

it follows that the renormalization of the coupling proceeds by uniform contraction of the target space sphere, as in equation (2) above with $a>0$. This is in accord with the celebrated result that the $O(N)$ sigma model becomes asymptotically free in the ultraviolet region $t \rightarrow-\infty$ when $N \geq 3$. Likewise, two dimensional sigma models with target spaces of negative constant curvature become asymptotically free in the infra-red region $t \rightarrow+\infty$, as they tend to expand according to equation (2) above with $a<0$. Also, when they flow backwards they develop a singularity as in the heat equation.

Next, let us consider more general sigma models which are defined on Riemannian manifolds $M$ using a local system of target space coordinates $\left\{X^{\mu} ; \mu=1,2, \cdots, n\right\}$ with metric $G_{\mu \nu}$. Using the two dimensional world-sheet metric $h_{i j}$ their action is

$$
S=\int d^{2} w \sqrt{\operatorname{det} h} h^{i j}\left(\partial_{i} X^{\mu}\right)\left(\partial_{j} X^{\nu}\right) G_{\mu \nu}
$$

In general the beta function is not zero and one finds within perturbation theory that the metric changes under world-sheet rescaling as [3]

$$
\Lambda^{-1} \frac{\partial}{\partial \Lambda^{-1}} G_{\mu \nu}=-\beta\left(G_{\mu \nu}\right)=-R_{\mu \nu}-\frac{1}{2} R_{\mu \rho \sigma \tau} R_{\nu}^{\rho \sigma \tau}+\cdots .
$$

Here, $R_{\mu \nu}$ summarizes the lowest order terms but there are also quadratic and higher curvature terms in the perturbative loop expansion. Again, setting $t=\log \Lambda^{-1}$, one can readily identify the Ricci flow (1) with the renormalization group equation of the target space metric by retaining only the lowest order term. The higher loop terms, as they 
are indicated above, can have an effect in regions where the curvature of the space grows large, for example close to the formation of singularities, but they will not be considered in the following. It should be noted nevertheless that the perturbative renormalization of sigma models to higher loops suggests the inclusion of specific higher curvature terms to the Ricci flow equation which might also be of interest to the mathematics community.

The Ricci flow equations admit a natural generalization when arbitrary reparametrizations of the target space coordinates $\delta X_{\mu}=-\xi_{\mu}$ are also included. They become

$$
\frac{\partial}{\partial t} G_{\mu \nu}=-R_{\mu \nu}+\nabla_{\mu} \xi_{\nu}+\nabla_{\nu} \xi_{\mu}
$$

where the vector field $\xi_{\mu}$ can depend on all target space coordinates as well as on time $t$. The role of reparametrizations along the flow is better understood by considering the renormalization group equations for a general background with metric $G_{\mu \nu}$ and dilaton field $\tilde{\Phi}$. Their general form in the sigma model frame is to lowest order

$$
\begin{aligned}
& \frac{\partial}{\partial t} G_{\mu \nu}=R_{\mu \nu}-2 \nabla_{\mu} \nabla_{\nu} \tilde{\Phi}+\nabla_{\mu} \xi_{\nu}+\nabla_{\nu} \xi_{\mu} \\
& \frac{\partial}{\partial t} \tilde{\Phi}=-(\nabla \tilde{\Phi})^{2}+\frac{1}{2} \nabla^{2} \tilde{\Phi}+\xi_{\mu} \nabla^{\mu} \tilde{\Phi}
\end{aligned}
$$

where arbitrary reparametrizations are also included. We observe that the effect of the dilaton is similar to reparametrizations generated by a gradient vector field $\xi_{\mu}$. In particular, choosing $\xi_{\mu}=\nabla_{\mu} \tilde{\Phi}$ the equations simplify to

$$
\frac{\partial}{\partial t} G_{\mu \nu}=-R_{\mu \nu}, \quad \frac{\partial}{\partial t} \tilde{\Phi}=\frac{1}{2} \nabla^{2} \tilde{\Phi}
$$

The first is the Ricci flow (1) and the second is an ordinary heat equation for $\tilde{\Phi}$. In general, reparametrizations assign non-trivial Weyl transformation laws to the target space coordinates which act as dilaton gradient in the renormalization group equations of two dimensional sigma models according to

$$
\delta_{\epsilon} h_{i j}=\epsilon h_{i j}, \quad \delta_{\epsilon} X^{\mu}=\epsilon \nabla^{\mu} \tilde{\Phi}
$$

Note in passing that adding the dilaton leads to interesting fixed point configurations of the beta function equations which are characterized by the condition $R_{\mu \nu}=2 \nabla_{\mu} \nabla_{\nu} \tilde{\Phi}$. A particularly simple solution in two dimensions is given in polar coordinates,

$$
d s^{2}=d r^{2}+\tanh ^{2} r d \theta^{2}, \quad \tilde{\Phi}(r)=\log (\cosh r),
$$

and describes the geometry of an infinitely long cigar with its tip located at $r=0$. This solution arose as model for two dimensional Euclidean black hole in the context of gauged WZW models, [4] (but see also [5]). Equivalently, it can be regarded as special instance of the original Ricci flow (1) by considering solutions where $G_{\mu \nu}(t)$ is the pull-back of an initial metric $G_{\mu \nu}(0)$ by a one-parameter family of diffeomorphisms generated by a 
vector field $\xi_{\mu}(t)$. These are self-similar solutions of the partial differential equation (1), called Ricci solitons, which satisfy the special condition

$$
R_{\mu \nu}=\nabla_{\mu} \xi_{\nu}+\nabla_{\nu} \xi_{\mu}
$$

as they move along by diffeomorphisms, [6]. They arose in the mathematics literature as limits of dilations of singularities of the Ricci flow. Thus, it is not surprising that the cigar configuration (12) was discovered independently in mathematics as first example of a steady gradient Ricci soliton with $\xi_{\mu}=\nabla_{\mu} \tilde{\Phi}$ as given above, [7]. It will also be relevant later in our discussion of axially symmetric deformations of the round sphere (sausage model) where the ultra-violet limit can be viewed as two such solitons glued together in their asymptotic region. Higher dimensional examples of rotational symmetric Ricci solitons also exist in the literature. Finally, it should be noted that Ricci solitons saturate the Harnack inequality and occupy a central role in the whole subject, [8].

In the mathematics literature one often considers the normalized Ricci flows as variant of (1). To motivate their definition let us consider the volume of the space $M$,

$$
V=\int_{M} \sqrt{\operatorname{det} G} d^{n} X
$$

Its change is controlled by the Einstein-Hilbert action, since the Ricci flow (1) implies

$$
\frac{\partial V}{\partial t}=\frac{1}{2} \int_{M} d^{n} X \sqrt{\operatorname{det} G} G^{\mu \nu} \frac{\partial G_{\mu \nu}}{\partial t}=-\frac{1}{2} \int_{M} d^{n} X \sqrt{\operatorname{det} G} R[G] .
$$

Thus the volume of a closed manifold is not preserved under the flow. It will decrease if the Ricci scalar curvature $R>0$ and increase if $R<0$, in agreement with the behavior of the simple solution (2) that describes deformations of spaces with constant curvature forward in time. Volume preserving deformations are defined by considering the normalized Ricci flow

$$
\frac{\partial}{\partial t} G_{\mu \nu}=-R_{\mu \nu}+\frac{1}{n} \bar{R} G_{\mu \nu}
$$

where $\bar{R}$ is the average (mean) scalar curvature of the manifold $M$,

$$
\bar{R}=\frac{1}{V} \int_{M} d^{n} X \sqrt{\operatorname{det} G} R[G]
$$

Since $\bar{R}$ is only a function of $t$, it follows that the fixed point solutions of (16) correspond to configurations of constant curvature.

The normalized flow follows from the unnormalized equation (1) by reparametrizing in time and rescaling the metric in space by a function of $t$. Note in this respect that the rescaling of the metric by an arbitrary function of $t, \tilde{G}_{\mu \nu}=f(t) G_{\mu \nu}$, does not affect the Ricci curvature tensor as $R_{\mu \nu}=\tilde{R}_{\mu \nu}$, but it transforms the unnormalized Ricci flow into the general form

$$
\frac{\partial}{\partial \tilde{t}} \tilde{G}_{\mu \nu}=-\tilde{R}_{\mu \nu}+\lambda(\tilde{t}) \tilde{G}_{\mu \nu}
$$


where the new time variable $\tilde{t}$ and the function $\lambda(\tilde{t})$ are determined as follows,

$$
\tilde{t}=\int d t f(t), \quad \lambda(\tilde{t})=\frac{f^{\prime}(t)}{f^{2}(t)} .
$$

Clearly, the normalized Ricci flow (16) corresponds to the choice $\lambda(t)=\bar{R} / n$ (after dropping the tilde in the notation) and has $\partial V / \partial t=0$. Again, the simple solution (2) is useful to understand the equivalence between the two flows by time rescaling.

The normalized deformation (16) has better chance to admit long-lived solutions compared to the unnormalized one whose solutions typically exist only for short time. Indeed, the uniformly contracting metrics (2) become extinct in finite time after reaching a singularity at $t=1 / a$, whereas their description as steady state solutions of the normalized Ricci flow allows them to exist for infinitely long time. Of course, this is not an issue for the case of uniformly expanding metrics as they exist for long time. The main task is to determine the conditions under which the solutions of the normalized Ricci flow exist for sufficiently long time and converge to canonical metrics, i.e., metrics of constant curvature in various forms. This is precisely the starting point for using the Ricci flow to explore the geometrization conjecture, but the results depend heavily on the number of dimensions. It is important in this respect to determine first the time evolution of the curvature. The Ricci flow equation (1) yields the following non-linear heat equation for the Ricci scalar curvature,

$$
\frac{\partial R}{\partial t}=\frac{1}{2} \nabla^{2} R+R_{\mu \nu} R^{\mu \nu}
$$

which in turn implies that positivity of $R$ is preserved on closed manifolds in any number of dimensions; negative scalar curvature is not preserved in general. On the other hand, the time evolution of the components of the Ricci curvature tensor is more complicated as it also involves the components of the Riemann curvature tensor and positivity is not necessarily maintained. In low dimensions the situation is expected to be more tractable simply because of numerology: in two dimensions the Ricci scalar curvature determines algebraically all the components of the Ricci as well as the Riemann curvature tensors, whereas in three dimensions the number of independent components of the Riemann curvature tensor is six and equals the number of independent components of the Ricci curvature tensor. In the following we focus our discussion to two and three dimensions and present a brief survey of the main results.

Let us first consider the Ricci flows on two dimensional manifolds $M^{2}$ which also extend easily to three dimensional geometries of the form $S^{1} \times M^{2}$. Closed surfaces are topologically classified by their Euler number given by the genus $g$,

$$
\chi(M)=\frac{1}{4 \pi} \int_{M} d^{2} X \sqrt{\operatorname{det} G} R[G]=2(1-g) .
$$

The classic uniformization theorem of Poincaré and Koebe describes their geometrization by asserting the existence of constant curvature metrics on $M$ whose form depends on whether the genus is 0,1 or $g \geq 2$ : in general they are quotients of $S^{2}, R^{2}$ or $H^{2}$ (with curvature $+1,0$ or -1 respectively) by a discrete subgroup $\Gamma$ acting freely and 
isometrically. The Ricci flow of a metric on $M$ depends crucially on the genus, since its volume changes at a constant rate,

$$
\frac{\partial V}{\partial t}=-2 \pi \chi(M)
$$

following equation (15) for the unnormalized Ricci flow. For $g=0$ there is contraction as in the simple solution (2) with $a>0$, for $g=1$ there is no deformation at all since the torus defines a conformally invariant sigma model, and for $g \geq 2$ there is expansion as in (2) with $a<0$. The normalized Ricci flow fixes the volume and it has been shown in all cases that the solutions converge to constant curvature metrics (depending on the genus) for any initial metric on $M,[7,9]$. Thus, the Ricci flows provide another proof of the uniformization theorem of closed two dimensional surfaces by new method. This is precisely the idea one tries to apply to higher (three) dimensional manifolds in favor of the geometrization conjecture.

The Ricci flow equation (1) also exhibits a very rich algebraic structure in two dimensions as outlined below. Its takes particularly simple form in a local system of conformally flat coordinates

$$
d s_{\mathrm{t}}^{2}=2 e^{\Phi\left(z_{+}, z_{-} ; t\right)} d z_{+} d z_{-} .
$$

Since the only non-vanishing component of the Ricci curvature is $R_{+-}=-\partial_{+} \partial_{-} \Phi$, we obtain the following non-linear differential equation for the conformal factor,

$$
\frac{\partial}{\partial t} e^{\Phi\left(z_{+}, z_{-}, ;\right)}=\partial_{+} \partial_{-} \Phi\left(z_{+}, z_{-} ; t\right) \text {. }
$$

This equation is integrable for it can be brought into zero curvature form using gauge connections that take values in the local part of an infinite dimensional algebra with Cartan kernel $K\left(t, t^{\prime}\right)=\partial_{t} \delta\left(t-t^{\prime}\right)$, [10]. This mathematical structure fits into the general class of continual Lie algebras by incorporating the deformation variable $t$ into the root system, but it has the peculiar feature that the Cartan kernel is antisymmetric. Equation (24) is actually the Toda system associated to this algebra and therefore its general solution can be expressed in terms of a one-parameter family of two dimensional free fields using the group theoretical formulae that are available in such cases.

More precisely, let us consider a Lie algebra with Cartan-Weyl generators that satisfy the commutation relations

$$
\begin{aligned}
& {\left[X^{+}(\varphi), X^{-}(\psi)\right]=H(\varphi \psi), \quad[H(\varphi), H(\psi)]=0,} \\
& {\left[H(\varphi), X^{ \pm}(\psi)\right]=\mp X^{ \pm}\left(\varphi^{\prime} \psi\right) .}
\end{aligned}
$$

Here, $\varphi$ and $\psi$ are functions of the continuous variable $t$ and prime denotes the derivative with respect to it. Equivalently, we may consider generators $X^{ \pm}(t)$ and $H(t)$ that depend on the continuous variable $t$ and write down their commutation relations using the Cartan kernel $K\left(t, t^{\prime}\right)=\partial_{t} \delta\left(t-t^{\prime}\right)$. We prefer to define the algebra by smearing the generators with arbitrary functions of $t$, as it is commonly done in the theory of distributions, in which case the Cartan operator is $K=d / d t$. Then, the zero curvature condition

$$
\left[\partial_{+}+A_{+}\left(z_{+}, z_{-}\right), \partial_{-}+A_{-}\left(z_{+}, z_{-}\right)\right]=0
$$


where $A_{ \pm}$take values in this infinite dimensional algebra with

$$
A_{+}=H(\Psi)+i X^{+}(1), \quad A_{-}=i X^{-}\left(e^{\Phi}\right)
$$

reads as follows,

$$
\partial_{-} \Psi=e^{\Phi}, \quad \partial_{+} \Phi=\partial_{t} \Psi
$$

Eliminating the variable $\Psi$ we arrive at the two dimensional Ricci flow (24) as advertised above.

The Toda field formulation of the Ricci flow allows for the construction of its general solution in terms of free fields by Bäcklund transformations. The group theoretical expressions that have been known for Toda systems associated to simple finite dimensional Lie algebras can be easily extended to the case of continual Lie algebras, [11, 12]. For this let us introduce (formally) a highest weight state

$$
X^{+}\left(t^{\prime}\right)|t>=0, \quad<t| X^{-}\left(t^{\prime}\right)=0, \quad H\left(t^{\prime}\right)\left|t>=\delta\left(t-t^{\prime}\right)\right| t>
$$

subject to the normalization $<t \mid t>=1$. Then, the general solution of the Toda field equation (24) which is associated to the continual Lie algebra with Cartan operator $K=d / d t$ takes the form $[10]$

$$
\Phi\left(z_{+}, z_{-} ; t\right)=\Phi_{0}\left(z_{+}, z_{-} ; t\right)+\partial_{t}\left(<t\left|M_{+}^{-1}\left(z_{+} ; t\right) M_{-}\left(z_{-} ; t\right)\right| t>\right)
$$

where $M_{ \pm}$are the path-ordered exponentials

$$
M_{ \pm}\left(z_{ \pm} ; t\right)=\mathcal{P}\left(i \int^{z_{ \pm}} d z_{ \pm}^{\prime} \int^{t} d t^{\prime} e^{f^{ \pm}\left(z_{ \pm}^{\prime} ; t^{\prime}\right)} X^{ \pm}\left(t^{\prime}\right)\right)
$$

$\Phi_{0}\left(z_{+}, z_{-} ; t\right)=f^{+}\left(z_{+} ; t\right)+f^{-}\left(z_{-} ; t\right)$ is a one-parameter family of two dimensional free fields with $\partial_{\mp} f^{ \pm}\left(z_{ \pm} ; t\right)=0$ for all $t$.

In practice, one obtains a formal power series solution around the free field configuration by expanding the path-ordered exponentials as

$$
\begin{aligned}
<t\left|M_{+}^{-1} M_{-}\right| t>=1+\sum_{m=1}^{\infty} \int^{z_{+}} d z_{1}^{+} \cdots \int^{z_{m-1}^{+}} d z_{m}^{+} \int^{z_{-}} d z_{1}^{-} \cdots \int^{z_{m-1}^{-}} d z_{m}^{-} \times \\
\times \int \prod_{i=1}^{m} d t_{i} \int \prod_{i=1}^{m} d t_{i}^{\prime} \exp f^{+}\left(z_{i}^{+} ; t_{i}\right) \exp f^{-}\left(z_{i}^{-} ; t_{i}^{\prime}\right) D_{t}^{\left\{t_{1}, t_{2}, \cdots, t_{m} ; t_{1}^{\prime}, t_{2}^{\prime}, \cdots, t_{m}^{\prime}\right\}}
\end{aligned}
$$

where

$$
D_{t}^{\left\{t_{1}, t_{2}, \cdots, t_{m} ; t_{1}^{\prime}, t_{2}^{\prime}, \cdots, t_{m}^{\prime}\right\}}=<t\left|X^{+}\left(t_{1}\right) X^{+}\left(t_{2}\right) \cdots X^{+}\left(t_{m}\right) X^{-}\left(t_{m}^{\prime}\right) \cdots X^{-}\left(t_{2}^{\prime}\right) X^{-}\left(t_{1}^{\prime}\right)\right| t>.
$$

The computation of these elements can be done recursively using the commutation relations of the Cartan-Weyl generators. The resulting terms encode all the information about the underlying Lie algebra of the Toda system.

A remarkable feature of this approach is the emergence of a new description of geometric deformations based on infinite dimensional algebras. The time variable $t$ of the Ricci 
flows assumes an intrinsic role in the structure of the infinite dimensional Lie algebra used in the zero curvature formulation of the problem. The gauge connections $A_{ \pm}\left(z_{+}, z_{-}\right)$take values in the local part $\mathcal{G}_{-1} \oplus \mathcal{G}_{0} \oplus \mathcal{G}_{+1}$ of a continual contragradient Lie algebra with Cartan operator $K=d / d t$, which is denoted by $\mathcal{G}(d / d t)$. Although the complete structure of the algebra is not needed for the present purposes, it will be interesting to construct all other elements and their commutation relations beyond the fundamental system of its Cartan-Weyl generators. The only known result so far is the exponential growth of the number of independent generators of the subspaces $\mathcal{G}_{n}=\left[\mathcal{G}_{n-1}, \mathcal{G}_{+1}\right]$ with $n>1$ and $\mathcal{G}_{n}=\left[\mathcal{G}_{n+1}, \mathcal{G}_{-1}\right]$ with $n<-1$, which are obtained by taking successive commutation relations of the elements $X^{ \pm} \in \mathcal{G}_{ \pm 1}$ as in all contragradient Lie algebras. In particular, if $d_{n}$ denotes the dimension of the subspaces $\mathcal{G}_{n}$ relative to $\mathcal{G}_{0}$ generated by the Cartan element $H$, it follows by induction that $d_{n}=2^{|n|-2}$ for all $|n| \geq 2$, [12]. Clearly, this is a rather exotic algebraic structure that calls for further attention in the future. It will be interesting to have the analogue of Serre relations for $\mathcal{G}(d / d t)$, construct representations, and examine the relevance of its exponential growth in the algebraic formulation of dynamical problems, such as the Ricci flow. It is also of independent interest in the theory of generalized Kac-Moody algebras with non-symmetrizable Cartan matrices. For now, we are only satisfied with its use for the integration of the two dimensional Ricci flow.

Next, we present some explicit examples of Ricci trajectories on two dimensional manifolds which have attracted considerable attention in the physics literature. They all have axial symmetry and represent mini-superspace solutions of a more general dynamical system defined in the space of all possible metrics. The existence of such special trajectories relies on the property of the Ricci flow to preserve all isometries of an initial metric. Thus, they can also be derived by elementary techniques by allowing the metric to depend on a small number of time dependent moduli, as in all consistent mini-superspace truncations. The resulting configurations are easier to visualize when written in proper coordinates than in conformally flat frame, but the change of variables needs to be compensated by a vector field $\xi_{\mu}$ that generates the necessary reparametrization along the flows. In this case we seek solutions of the modified Ricci flow equations (8), although equally well we can describe them using the initial system (1) in conformally flat coordinates. It should be noted that all the examples we present in the following admit a free field realization according to the general solution of the corresponding Toda field equation given above; we refer the reader to the literature for further details, [10]. Such comparison is also useful to demonstrate the validity and convergence of the infinite power series expansion (32), which is only formally defined for the case of infinite dimensional algebras.

The sausage model provides the simplest non-trivial example of geometric deformations of compact spaces with spherical topology, $S^{2},[13]$. The solution is described in proper coordinates using the Jacobi elliptic function,

$$
d s_{\mathrm{t}}^{2}=\frac{k}{\gamma}\left(d u^{2}+\operatorname{sn}^{2}(u ; k) d \phi^{2}\right) \quad \text { with } k=\tanh (-\gamma t)
$$

where $0 \leq u \leq 2 K(k), \gamma \geq 0$ is an arbitrary constant that parametrizes the family of trajectories and $0 \leq \phi \leq 2 \pi$, [10]. The modulus $k$ runs from 1 to 0 as $t$ ranges from 
$-\infty$ to 0 and $K(k)$ denotes the complete elliptic integral of the first kind. There is also a corresponding gradient vector field $\xi_{u}(u)$ that compensates the change of coordinates from the conformally flat frame to (34) so that the deformation is consistently described by equation (8). When $\gamma=0$, we have $k=0$ in which case $\operatorname{sn}(u ; 0)=\sin u$. Since $k / \gamma=-t$, the metric flows as $d s_{\mathrm{t}}^{2}=-t\left(d u^{2}+\sin ^{2} u d \phi^{2}\right)$ with the angular variable $u$ ranging from 0 to $2 K(0)=\pi$. This particular solution describes a round sphere with linearly diminishing scale as $t$ runs from the ultra-violet region $t \rightarrow-\infty$ to the big crunch point $t=0$; it coincides with the simple solution (2) with $a>0$ for $n=2$ that dictates the running coupling of the $O(3)$ sigma model. When $\gamma>0$, the solution describes more general axially symmetric deformations of the sphere that look like sausages with characteristic size $2 K(k)$ given by the periodicity of the function $\operatorname{sn}^{2}(u ; k)$. In the ultraviolet limit $k \rightarrow 1$ the sausage is infinitely long becoming a cylinder $R \times S^{1}$ of radius $1 / \sqrt{\gamma}$. It can be alternatively viewed as a configuration two semi-infinite cigars glued together in their asymptotic region, since $\operatorname{sn}(u ; 1)=\tanh u$ and the metric around the tips assumes the form (12). The sausage tends to diminish in size when $k$ decreases until it shrinks to a point when $k=0$ for all values of the parameter $\gamma$.

Another solution in two dimensions that describes axially symmetric deformations of a negatively curved hyperboloid is given in proper coordinates

$$
d s_{\mathrm{t}}^{2}=\frac{k^{\prime 2}}{\gamma}\left(d u^{2}+\frac{1}{\operatorname{dn}^{2}(u ; k)} d \phi^{2}\right) \quad \text { with } k=\frac{1}{\cosh (\gamma t)}
$$

where $-K(k) \leq u \leq K(k), \gamma \geq 0$ and $0 \leq \phi \leq 2 \pi$, [10]. The modulus $k$ ranges from 1 to 0 as $t$ varies from 0 to the infra-red limit $t \rightarrow+\infty$. There is also a gradient vector field $\xi_{u}(u)$ to compensate the change of coordinates, as before. When $k=1$, the Jacobi elliptic function $\operatorname{dn}(u ; k)$ equals $1 / \cosh u$ and the geometry looks like an infinite negatively curved hyperboloid. When $k=0$, we have $\operatorname{dn}(u ; 0)=1$ and the geometry looks like a segment of flat cylinder of radius $1 / \sqrt{\gamma}$ as $u$ ranges from $-\pi / 2$ to $\pi / 2$. The special trajectory with $\gamma=0$ corresponds to the linear rescaling of the metric (2) with $a<0$, which leads to uniform expansion. However, this cannot be readily seen from the form of the metric (35) unless a factor $k^{\prime}$ is appropriately absorbed and the remaining scale of the line element becomes $k^{\prime} / \gamma=t$.

In the same context we can also consider deformations of the Poincaré metric on the upper half-plane $H^{2}$ which are described by the simple family of trajectories

$$
e^{\Phi\left(z_{+}, z_{-} ; t\right)}=\frac{2 t}{\gamma^{2} t^{2}+\left(z_{+}+z_{-}\right)^{2}}
$$

in conformally flat frame, [10]. For $\gamma=0$ the solution describes a uniform linear expansion of the standard Poincaré metric $d z_{+} d z_{-} /\left(z_{+}+z_{-}\right)^{2}$, as in equation (2), since the curvature is negative. For $\gamma \neq 0$ the deformation is more drastic and it is better visualized using proper coordinates. It will be interesting to have explicit examples of geometric deformations of $H^{2} / \Gamma$ for appropriate choices of discrete subgroups $\Gamma \in S L(2, Z)$ that correspond to higher genus Riemann surfaces. 
Finally, we consider the simplest example of a two dimensional non-compact space with initial curvature singularity (localized at the tip of a cone $C / Z_{n}$ ) which dissipates away after infinitely long time, [14]. It can be regarded as a fundamental solution of the non-linear equation (20) for the scalar curvature, which generalizes the Gaussian solution for the linear heat equation $\partial_{t} R=\nabla^{2} R / 2$ on the plane. The solution is best described in the frame

$$
d s_{\mathrm{t}}^{2}=f^{2}(r ; t) d r^{2}+r^{2} d \phi^{2}, \quad \xi_{r}=\frac{1}{2 t} r f(1-f), \quad \xi_{\phi}=0
$$

where $0 \leq \phi \leq 2 \pi / n$. The modified Ricci flow equation determines the form of the shape function $f(r ; t)$,

$$
\left(\frac{1}{f}-1\right) \exp \left(\frac{1}{f}-1\right)=(n-1) \exp \left(n-1-\frac{r^{2}}{2 t}\right) .
$$

When $t \rightarrow 0^{+}$we have $f \rightarrow 1$, which corresponds to the metric $d s^{2}=d r^{2}+r^{2} d \phi^{2}$ of a cone with opening angle $2 \pi / n$. On the other hand, the infra-red limit $t \rightarrow+\infty$ yields $f \rightarrow 1 / n$, which corresponds to the metric $d s^{2}=d r^{2}+r^{2} d \tilde{\phi}^{2}$ of the two dimensional plane in polar coordinates with $0 \leq \tilde{\phi} \leq 2 \pi$. The decay of a cone $C / Z_{n}$ to the plane $R^{2} \simeq C$ has paramount importance to the problem of (localized) tachyon condensation in closed string theory since the ten dimensional string vacuum $C / Z_{n} \oplus R^{7,1}$ has tachyonic states in the twisted sector of the conical block. They induce transitions to more stable vacua by reducing the order of the singularity, which eventually becomes completely resolved in the infra-red limit of the renormalization group flow. There are generalizations of this phenomenon to more complicated backgrounds that exhibit tachyons, but no explicit solutions are known for their decay patterns.

The final topic in this presentation is the application of Ricci flows to three dimensional geometries on closed Riemannian manifolds (see, for instance, [15] for an excellent exposition and [16] for comprehensive reviews of the latest mathematical developments). Positivity of the Ricci scalar curvature is maintained throughout the deformation according to equation (20). The same is true for the Ricci curvature tensor in three dimensions although this is not generally valid in higher dimensional spaces with $n>3$. Furthermore, it has been shown by Hamilton that any initial metric in three dimensions with everywhere positive definite Ricci curvature tensor evolves under the Ricci flow so that the manifold becomes rounder and rounder as it shrinks. If the metric is rescaled so that the volume remains constant, the corresponding solutions of the normalized Ricci flow will exist for infinitely long time and they all converge towards the constant curvature metric on $S^{3} / \Gamma$, where $\Gamma$ is a finite subgroup of $S O(4)$ acting freely on $S^{3}$, [17]. This space-form theorem geometrizes 3-manifolds of positive Ricci curvature via the Ricci flow and paves the way towards the proof of Thurston's geometrization conjecture which asserts that every component of the sphere and torus decomposition of any closed oriented 3-manifold admits a geometric structure. It includes as special case the Poincaré conjecture stating that a closed 3-manifold with trivial fundamental group must be homeomorphic to the 3-sphere; more generally, a closed 3-manifold with finite fundamental group must admit a metric of constant positive curvature (see, for instance, [16]). 
Further progress relies on the study of Ricci flows on more general 3-manifolds. The situation is more complicated when the Ricci scalar curvature is positive but the components of the Ricci curvature tensor are not all positive definite everywhere. The Ricci flow does not preserve negative Ricci curvature in dimensions $n \geq 3$. If at a generic point there are directions of positive as well as negative Ricci curvature, the flow will tend to contract or expand the metric locally. In general, such uneven geometric deformations soon run into singularities, which often can not be avoided even by the normalized Ricci flow. A typical example of curvature singularity forming in the normalized Ricci flow is provided by the dumb-bell geometry that consists of two spherical regions joined together by a suitably long throat. Even if the three-dimensional volume is preserved, the throat will have the tendency to pinch as it is topologically described by an interval times $S^{2}$ and the positive curvature of $S^{2}$ overtakes the small but negative curvature in the third direction. Another example is provided by positive scalar curvature metrics on a connected sum of $S^{3} / \Gamma$ and $S^{1} \times S^{2}$ which develop singularities as their deformations can not possibly converge to a round metric. On the other hand, there are non-singular solutions of the normalized Ricci flow which exist for all time, as in the case of a spherical space form. The topological classification of closed manifolds which admit non-singular solutions of the normalized Ricci flow is now well understood in three dimensions, [18]. The more difficult part is the study of singularities and the application of geometric surgeries before they occur so that the solutions can be continued separately on different components until eventually they converge to constant curvature metrics.

The analysis of singularities is quite intricate and it will not be discussed in detail (see, however, [19]). We only note here that one way in which singularities may arise along the Ricci flow is that a 2 -sphere in the three dimensional manifold may collapse to a point in finite time. Then, one should perform surgery analogous to the decomposition of a general 3 -manifold into connected sum of prime factors by cutting the space along $S^{2}$ and gluing 3-balls on the individual components. After a finite number of such surgeries it turns out that the Ricci flow on a closed manifold $M$ leads to the topological decomposition

$$
M=K_{1} \# \cdots \# K_{n} \#\left(S^{3} / \Gamma_{1}\right) \# \cdots \#\left(S^{3} / \Gamma_{m}\right) \#\left(S^{1} \times S^{2}\right)_{1} \# \cdots \#\left(S^{1} \times S^{2}\right)_{r} .
$$

The factors $S^{3} / \Gamma_{i}$ and $S^{1} \times S^{2}$ can be disregarded as they become extinct in finite time under the unnormalized Ricci flow. The remaining factors $K_{i}$ exist for sufficiently long time and each one can be decomposed further as union of a complete hyperbolic manifold of finite volume and a graph manifold along a collection of incompressible embedded tori. Further decomposition of graph manifolds into Seifert fibered components is quite standard in topology; they split by disjoint embedded tori into pieces, each of which is a circle bundle over a surface. The recent advances towards the proof of the geometrization conjecture in three dimensions are due to Perelman, [20].

This result is analogous to the uniformization of closed surfaces in two dimensions. However, unlike two dimensions, the solutions of the normalized Ricci flow on 3-manifolds do not always exist for infinitely long time. This fact is attributed to the existence of essential 2-spheres, i.e., embedded 2-spheres that do not always bound a 3-ball in three 
dimensions and which tend to collapse in finite time. Put differently, a general 3-manifold is not geometric for any Riemannian metric on it can have degenerate regions. Thurston's geometrization conjecture only applies to each irreducible component of the sphere and torus decomposition of a 3-manifold and in that sense the Ricci flows have to be supplemented by surgery prior to the formation of singularities. Thus, the renormalization group properties of the $O(3)$ sigma model are responsible for the appearance of singularities in three dimensions caused by the collapse of essential $S^{2}$-cycles. In two dimensions, a similar decomposition of a higher genus Riemann surface into connected sum of tori is done by cutting along $S^{1}$-cycles which are incompressible under the Ricci flow, and as a result the corresponding solutions can never run into singularities. Finally, similar considerations can also apply to higher dimensions $n>3$, but the behavior of Ricci flows and the formation patterns of singularities are more complicated.

Summarizing, the Ricci flows in low dimensions have very rich algebraic and geometric properties which should be explored further. It still remains to understand the deeper role of the infinite dimensional Lie algebra $\mathcal{G}(d / d t)$ used in the formulation of two dimensional geometric deformations as integrable system. This algebra also dictates the behavior of Ricci flow in higher dimensions through the geometric deformations of two dimensional embedded submanifolds. Various entropy formulae for the Ricci flow and their relation to Zamolodchikov's $C$-theorem, [21], should also be examined in this context. Finally, the problem of tachyon condensation in closed string theory and its relation to the geometrization program of Riemannian manifolds via the Ricci flow in the weak gravitational regime should be investigated in more general terms.

We also note for completeness that the two dimensional Ricci flow equation (24) arises in other areas of physics where superfast diffusion processes take place. First, it appears in studies of the central limit approximation to Carleman's model of the Boltzmann equation, [22]. Second, it describes the cross-field convective diffusion of plasma including mirror effects, [23]. Third, it governs the expansion of a thermalized electron cloud described by isothermal Maxwell distribution, [24], where the solution (36) is also found. Finally, it appears as limiting case of the porous medium equation (see, for instance, [25] and references therein). The cigar soliton (12) coincides with the so-called Barenblatt solution in the theory of porous media, [26], whereas the sausage deformation of the round sphere $[13,10]$ coincides with the axi-symmetric solution found in [27] when written in conformally flat frame.

\section{Acknowledgments}

This work was supported in part by the European Research and Training Networks "Superstring Theory" (HPRN-CT-2000-00122) and "Quantum Structure of Spacetime" (HPRN-CT-2000-00131), as well as NATO Collaborative Linkage Grant "Algebraic and Geometric Aspects of Conformal Field Theories and Superstrings" (PST.CLG.978785). I am also grateful to the organizers of the conference for their kind invitation and generous financial support. Special thanks are due to the French string community for creating a very stimulating environment in Paris in the summer 2004. 


\section{References}

[1] H.-D. Cao, B. Chow, S.-C. Chu and S.-T. Yau eds, "Collected papers on Ricci flow", International Press, Somerville, 2003.

[2] A.M. Polyakov, "Gauge Fields and Strings", Contemporary Concepts in Physics, Harwood Academic Publishers, Chur, 1987.

[3] D. Friedan, "Non-linear models in $2+\epsilon$ dimensions", Ann. Phys. 163 (1985) 318.

[4] E. Witten, "String theory and black holes", Phys. Rev. D44 (1991) 314.

[5] G. Mandal, A. Sengupta and S. Wadia, "Classical solutions of two-dimensional string theory", Mod. Phys. Lett. A6 (1991) 1685; S. Elitzur, A. Forge and E. Rabinovici, "Some global aspects of string compactifications", Nucl. Phys. B359 (1991) 581.

[6] R. Hamilton, "Eternal solutions solutions to the Ricci flow", J. Diff. Geom. 38 (1993) 1.

[7] R. Hamilton, "The Ricci flow on surfaces", Contemp. Math. $\underline{71}$ (1988) 237.

[8] R. Hamilton, "The Harnack estimate for the Ricci flow", J. Diff. Geom. 37 (1993) 225.

[9] B. Chow, "The Ricci flow on the 2-sphere", J. Diff. Geom. 33 (1991) 325; J. Bartz, M. Struwe and R. Ye, "A new approach to the Ricci flow on $S^{2}$ ", Ann. Sc. Norm. Super. Pissa Cl. Sci. (4) 21 (1994) 475; R. Hamilton, "An isoperimetric estimate for the Ricci flow on surfaces", in Modern Methods in Complex Analysis, eds T. Bloom et al, Ann. Math. Studies, vol. 137, Princeton University Press, Princeton, 1995.

[10] I. Bakas, "Renormalization group flows and continual Lie algebras", JHEP $\underline{0308}$ (2003) 013.

[11] M. Saveliev, "Integro-differential non-linear equations and continual Lie algebras", Commun. Math. Phys. 121 (1989) 283.

[12] M. Saveliev and A. Vershik, "Continual analogs of contragradient Lie algebras (Lie algebras with a Cartan operator and non-linear dynamical systems), Commun. Math. Phys. $\underline{126}$ (1989) 367.

[13] V.A. Fateev, E. Onofri and Al.B. Zamolodchikov, "Integrable deformations of the O(3) sigma model. The sausage model", Nucl. Phys. B406 [FS] (1993) 521.

[14] A. Adams, J. Polchinski and E. Silverstein, "Don't panic! Closed string tachyons in ALE space-times", JHEP $\underline{0110}$ (2001) 029; M. Gutperle, M. Headrick, S. Minwalla and V. Schomerus, "Space-time energy decreases under world-sheet RG flow", JHEP $\underline{0301}(2003) 073$. 
[15] H.-D. Cao and B. Chow, "Recent developments on the Ricci flow", Bull. Amer. Math. Soc. 36 (1999) 59; B. Chow and D. Knopf, "The Ricci Flow: An Introduction", Mathematical Surveys and Monographs, vol. 110, American Mathematical Society, Providence, 2004.

[16] J. Milnor, "Towards the Poincaré conjecture and the classification of 3-manifolds", Notices Amer. Math. Soc. 50 (2003) 1226; M. Anderson, "Geometrization of 3manifolds via the Ricci flow", Notices Amer. Math, Soc. $\underline{51}$ (2004) 184.

[17] R. Hamilton, "Three-manifolds with positive Ricci curvature", J. Diff. Geom. $\underline{17}$ (1982) 255.

[18] R. Hamilton, "Non-singular solutions of the Ricci flows on three-manifolds", Comm. Anal. Geom. $\underline{7}$ (1999) 695.

[19] R. Hamilton, "Formation of singularities in the Ricci flow", Surveys in Diff. Geom. $\underline{2}(1995) 7$.

[20] G. Perelman, "The entropy formula for the Ricci flow and its geometric applications", preprint, math.DG/0211159; "Ricci flow with surgery on three-manifolds", preprint, math.DG/0303109; "Finite extinction time for the solutions to the Ricci flow on certain three-manifolds", preprint, math.DG/0307245.

[21] A.B. Zamolodchikov, "Irreversibility of the flux of the renormalization group in a 2D field theory", JETP Lett. $\underline{43}$ (1986) 730.

[22] T. Kurtz, "Convergence of sequences of semigroups of non-linear operators with an application to gas kinetics", Trans. Amer. Math. Soc. 186 (1973) 259; H. McKean, "The central limit theorem for Carleman's equation", Israel J. Math. $\underline{21}$ (1975) 54.

[23] T. Kamimura and J. Dawson, "Effect of mirroring on convective transport in plasmas", Phys. Rev. Lett. $\underline{36}$ (1976) 313; J. Drake and J. Berryman, "Theory of nonlinear diffusion of plasma across the magnetic field of a toroidal multipole", Phys. Fluids, 20 (1977) 851; J. Berryman and C. Holland, "Asymptotic behavior of the non-linear diffusion equation $n_{\mathrm{t}}=\left(n^{-1} n_{\mathrm{x}}\right)_{\mathrm{x}}$ ", J. Math. Phys. $\underline{23}$ (1982) 983.

[24] K. Lonngren and A. Hirose, "Expansion of an electron cloud", Phys. Lett. A59 (1976) 285.

[25] L.-F. Wu, "A new result for the porous medium equation derived from the Ricci flow", Bull. Amer. Math. Soc. $\underline{28}$ (1993) 90.

[26] D. Aronson, "The porous medium equations", in Some Problems in Non-linear Diffusion, eds A. Fasano et al, Lect. Notes Math., vol. 1224, Springer, New York, 1986.

[27] P. Rosenau, "Fast and superfast diffusion processes", Phys. Rev. Lett. $\underline{74}$ (1995) 1056. 